\title{
Selenium bio-fortification: an alternative to improve phytochemicals and bioactivities of plant foods
}

\section{Rattanamanee Chomchan ${ }^{1}$, Sunisa Siripongvutikorn², and Panupong Puttarak ${ }^{3}$}

${ }^{1}$ Interdisciplinary Graduated School of Nutraceutical and Functional Food, Prince of Songkla University, Hat-Yai, Songkla 90112, Thailand; ${ }^{2}$ Department of Food Technology, Faculty of Agro-Industry, Prince of Songkla University, Hat-Yai, Songkla 90112, Thailand; ${ }^{3}$ Department of Pharmacognosy and Pharmaceutical Botany, Faculty of Pharmaceutical Sciences, Prince of Songkla University, Hat-Yai, Songkhla 90112, Thailand

Corresponding author: Sunisa Siripongvutikorn, $\mathrm{PhD}$, Department of Food Technology, Faculty of Agro-Industry, Prince of Songkla University, Hat-Yai, Songkla 90112, Thailand

Submission Date: January $8^{\text {th }}$, 2017, Acceptance Date: April $23^{\text {rd }}, 2017$, Publication Date: April $30^{\text {th }}, 2017$

Citation: Chomchan R., Siripongvutikorn S., Puttarak P. Selenium bio-fortification: an alternative to improve phytochemicals and bioactivities of plant foods. Functional Foods in Health and Disease 2017; 7(4); 263-279

\begin{abstract}
Plants are major food sources which possess marvelous health benefits to human beings. To the present age, the effort to improve composites in plants using a bio-fortification technique is expedient to obtain super plant foods. Selenium (Se) is an essential trace element that works on antioxidant system in living beings. Plants have been bio-fortified with Se worldwide to increase Se content in addition to improving the bioactive compounds and biological properties. However, restricted documents were elucidated on the summary and underlying mechanisms which provide beneficial effects on plant phytochemicals and bioactivities improvement. Accordingly, this review article attempts to study available literature on Se bio-fortified plants while also clarifying possible routes through which Se may affect plants biochemical process. Increasing the content of Se bio-fortified in plants resulted in both positive and negative effects. The appropriate exogenous concentration of Se bio-fortification varies among the individual plants as different species have the ability to accumulate and tolerate different levels of Se. The concentration of Se which can support growth or induce them to the early stage of stress are strategic to provide desired effects on an improvement of phytochemicals. Moreover, Se bio-fortified molecules acts as a protectant against drought, cold, UV, heavy metals, and salt strains. The greater antioxidant, anti-cancer, and prebiotic properties were also reported from an increased amount of phytochemical and the synergistic effects of Se and plant phytochemicals. However, higher doses of Se fortified were
\end{abstract}


toxic to plants at specific levels. Consequently, Se bio-fortification can be claimed as a unique strategy to improve plant treasures.

Keywords: Selenium, Bio-fortification, Phytochemicals, Bioactivities

\section{BACKGROUND:}

Plant based diets have long been known as the dominant source of fuel for living things. Nowadays, plant based diets have received more attention as contemporary education evidence delivered the data on their marvelous health benefits and to promote the world population's well-being. The magnificent non-nutrient compounds which creates valuables to them were called "phytochemical". The word "phytochemical" refers to the chemicals that are produced naturally in a plant as secondary metabolites [1]. They are also referred to as the words "nutraceutical" or "phyto-protectant". Though phytochemicals are not crucial for the survival of plants, they are essential in promoting good health and supporting plant development throughout their life cycle. Accordingly, literature and evidence from epidemiological studies have been intensely investigated on the properties of these phytochemicals which reveal the favorable bioactivities on human health when consumed as sources of antioxidant, anti-inflammatory, anti-carcinogenic, anti-diabetic, and anti-microbial constituents which further defend against many chronic diseases including cardiovascular diseases, cancer, diabetes, hypertension, and obesity [2-4].

As the compounds of a plant-based diet have been receiving substantial interest, the strategies on how to improve those phytochemicals production in plants to promote plant nutritional values are more significant and relevant. Elicitation techniques have been applied onto plants as one of the good strategies to induce the production of phytochemical compounds using biotic elicitors including protein [5], oligosaccharides, polysaccharides [6], and abiotic elicitors such as physical stress [7]. Bio-fortification or mineral elicitation is the term that has been proposed as the process of increasing micronutrients into plant during plant growth [8]. It can be applied using the traditional agronomic technology such as the use of micronutrients fertilizers in soil and solution or the foliar application [9]. This term can also be referred to as "supplementation," "enrichment," or "fertilization" with the same denotation in this area of work. Accompanied by global mineral deficiency problems, bio-fortification is an approaching strategy which primarily emerges to deal with mineral deficiency problems. Bio-fortification can provide an accumulation of plant micronutrients as well as additional extraordinarily outgrowth to induce plants' secondary metabolites production. The biosynthesis of many secondary metabolites in plants is a defense mechanism of plants to equilibrate their system from stressors. Minerals applied can possibly induce plant to stress, thereafter plants may react by producing larger quantity of enzymes and phytochemical compounds to handle themselves from these injurious conditions. Moreover, minerals may play role as important cofactor of enzymes which is used for normal growth and the manufacture of primary and secondary metabolites. Consequently, they can be substrates and cofactors for these compounds production. Therefore, plant bio-fortification is one of the methods which gain increased quantity of minerals in plants, in addition to being a fascinating approach for promoting beneficial phytochemical compounds and finally providing great biological properties to plants. Selenium (Se) is the mineral responsible for antioxidant protection. The use of this mineral bio-fortification has been spread worldwide. However, restricted documents were 
elucidated on the summary in addition to underlying mechanisms that affect plant phytochemicals and bioactivities improvement. Therefore, this review aimed to evaluate available literature on Se bio-fortified plants and explain conceivable paths of action which reflect changes of phytochemical contents and bioactivities of various plants.

\section{SELENIUM IN PLANT METABOLISM}

Se is an essential trace element required by human, animals, microorganisms, and plants [10]. It originally enters the food chain through plants, although typical Se content of food varies from very excellent source in seafood, meat, poultry, and grains. Se content in plants is greatly influenced by the amount of Se in soil which varies around the world [11]. Additionally, the uptake and accumulation of Se also depends on many factors including the ability of plant species to absorb and metabolize Se, the form of use, and atmosphere conditions including $\mathrm{pH}$ and the presence of ion [12]. Three different groups of plants separated by the ability to uptake Se in plant tissues are non-accumulator, secondary accumulator, and primary accumulator which accumulate less than $25 \mathrm{mg} \mathrm{Se} / \mathrm{kg}$ dry weight, 25 to $100 \mathrm{mg} \mathrm{Se} / \mathrm{kg}$ dry weight and 100 to $10,000 \mathrm{mg} \mathrm{Se} / \mathrm{kg}$ dry weight respectively. A schematic of Se metabolism in plants is illustrated in Figure 1.

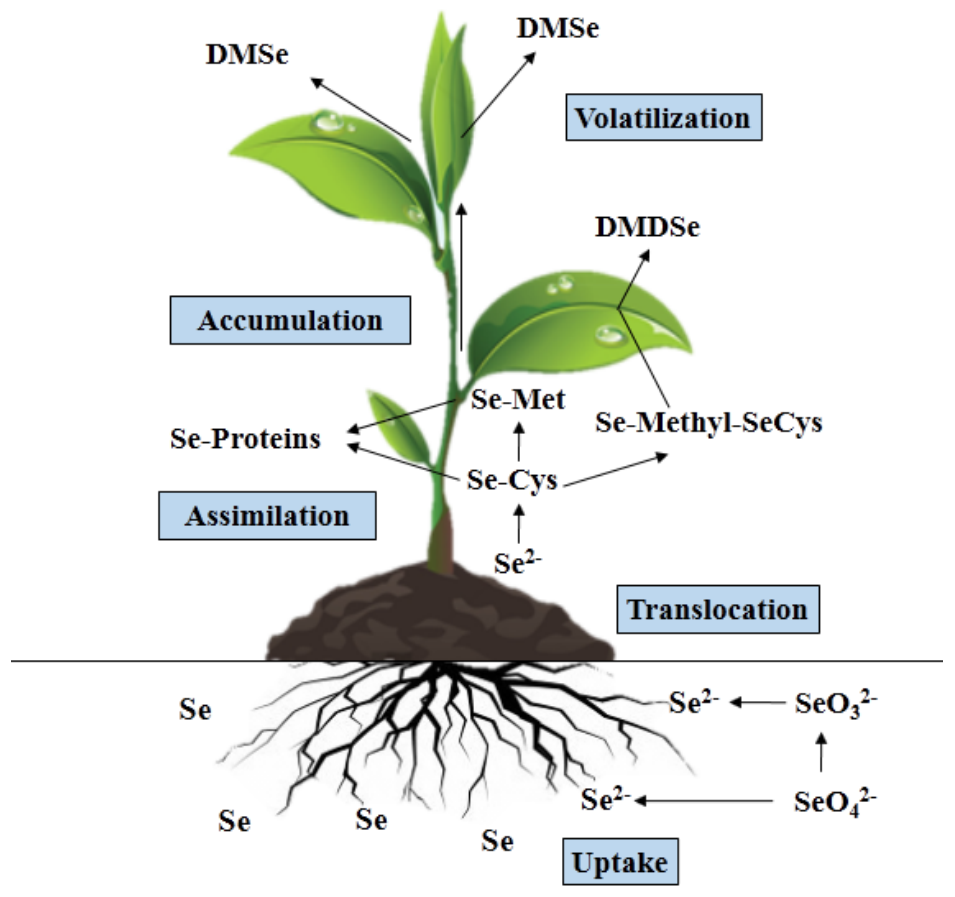

Figure 1. The schematic of Se metabolism in plants. Abbreviations: Se-Proteins, seleno-proteins; Se-Met, seleno-methionine; Se-Cys, seleno-cysteine; Se-Methyl-SeCys, seleno-methylselenocysteine; DMSe, dimethyl-selenide; DMDSe, dimethyl-diselenide [11].

Se is primarily taken up by plants as inorganic selenate $\left(\mathrm{SeO}_{4}{ }^{2-}\right)$ or selenite $\left(\mathrm{SeO}_{3}{ }^{2-}\right)$ [13] While plants absorb Se through roots, it incorporates plant proteins to form seleno-cystein (SeCys) and seleno-methionine (Se-Met) at usual levels. As the chemical and physical properties of Se and sulfur (S) are closely related, Se is taken up by plants in the similar way to that of S via sulfate transporter in the root plasma membrane. Therefore, both atoms are competitive candidates 
for the same binding sites and showed the antagonistic relationship [14]. Furthermore, plants have the biochemical processes which can get rid of excess Se to volatile Se compounds in the form of dimethyl-selenide (DMSe) or dimethyl-diselenide (DMDSe) by a process called volatilization which is also recognized as a detoxification process [15].

\section{SELENIUM BIO-FORTIFICATION APPROACH}

Essentially, Se has been recognized as an important trace element in antioxidant defense system in all living beings. It works on protecting the host against free radicals and destruction through being a core part of primary antioxidant enzymes named glutathione peroxidase (GPx). Moreover, Se is also a part of other seleno-proteins which have various functional roles in human body including Thioredoxin reductases which regulate DNA synthesis and gene expression. Iodothyronine deiodinases, which regulates thyroid hormone and Selenoprotein $\mathrm{S}$, is also an important factor for anti-inflammation and protecting cells from stress-induced apoptosis [12]. For plants, voluminous advantageous roles exerted by Se have also been reported that encompass supporting growth rate, stimulating seed germination, enhancing yield, and delaying senescence, while increasing plant respiratory potential and accumulation of metabolites in addition to manipulating plant homeostasis in stress conditions $[16,17]$. However, only the proper amount of Se demonstrated positive effects while higher amounts can lead to toxicity. The effort of Se biofortification began in Finland since the 1980s to successfully increase Se content in vegetables crops [18]. Afterwards, this technique became worthwhile to many deficient regions throughout the world. Nowadays, besides the need for an improvement of Se content, new challenges also include concerns about the promotion of phytochemicals in plants using Se bio-fortification due to various biological properties employed by these phytochemical components, which thereby may encourage positive effects to human health. To date, large number of studies were interested in this issue and reported effects on the influence of Se bio-fortification to expedient compounds level and bioactivities in numerous plants $[16,17,18]$. Main groups of Se bio-fortified plants were in Se accumulating plants such as broccoli, onion, garlic, and chives due to their high accumulation ability in addition to grains such as wheat, rice, rye, and barley attributable to their function as a main energy source for world population. Furthermore, there has been significant work in supplying common vegetables, including lettuce, cabbage, tomato, and potato. Sodium selenate and sodium selenite were the main forms applied to plants, as they are naturally occurring in the environment. Selenate was highly absorbable than selenite [19], yet the cost was twofold. Se can stimulate the production of phytochemicals due to many different procedures applied, seed priming, foliar application or grown in soil, any supporting materials or even grown in hydroponic solution system.

\section{INFLUENCE OF SELENIUM ON PLANT PHYTOCHEMICALS Chlorophyll}

Chlorophyll is the foremost component which works on energy manufacturing for plants through a process called photosynthesis which also provides green color to the plants [20]. It is correlated to the growth of plants as every living being needs energy for the progression of life. Consequently, any factors that have undesirable effects on plants are also likely to harm the production of this photosynthetic compound. Se has been positively reported to affect the production chlorophyll in 
many plants. Precise outcomes from the supplementation of sodium selenite to goji berry leaves (Lycium chinense) up to $5 \mathrm{mg} \mathrm{Se} / \mathrm{L}$ by Dong et al. (2013) showed an enhancement of $229 \%$ chlorophyll a and 205\% chlorophyll b contents compared to the control [21]. Similar results have also been reported in kenaf [22] and lettuce [23, 24]. It can be suggested that at low concentrations, Se may promote proper conditions to plants and influence the efficient flow of electrons in the respiratory chain, which is related to the rate of plant photosynthesis and promotion of chlorophyll biosynthesis. Furthermore, another reason indicated by a study conducted by Padmaja et al. (1990) is that Se possesses a role as a novel regulator of porphyrin biosynthesis by controlling the porphobilinogen synthase activity [25]. Consequently, Se is important for the biosynthesis of chlorophyll. Moreover, Se also exerted an improvement in the photosynthetic rate in plants exposed to many stress conditions. For example, this includes the improvement of chlorophyll content in wheat seedling grown during drought stress [26], cold stress [27], and potatoes grown under light stress [28] by eliminating radical disturbances and rebalance plant cells system. However, Se treatment at higher levels can cause an adverse effect to plants by increasing the rate of lipid peroxidation and inhibiting porphobilinogen synthase activity, which decreases chlorophyll content. For example, Se treatment at higher levels can decrease in chlorophyll content in mung bean seedlings [29] and basil plants [30], and can also eventually lead to death. Nevertheless, the chlorophyll content in plant varies not only from the direct effect of Se but also from its indirect effect, mostly through its influence to other compounds including phenolic compounds. The level of phenolic compounds will probably be increased by Se as a stressor, while also further regulating a reduction in chlorophyll biosynthesis due to the competitive effects between polyphenol and chlorophyll biosynthesis [31].

\section{Carotenoids}

Carotenoids are the yellow, orange, or red pigment found in the chloroplast of plants and living photosynthetic cells. They serve two vital roles in plants, the first being the absorption of light energy for photosynthesis process and secondly protecting chlorophyll from photo-damage and harmful interferences such as anti-oxidative substances [32]. They can be divided into two classes which are xanthophylls (oxygen containing carotenoids) and carotenes (purely hydrocarbons carotenoids). Well known xanthophylls are named as follows, lutein and zeaxanthin, while carotenes group includes $\alpha$-carotene, $\beta$-carotene, and lycopene. Plants carotenoid contents were reported to be affected by Se bio-fortification in many studies since Se addition at some levels can induce plant to stress conditions. Basil plants fortified with high Se levels (30-120 mg Se/L) exhibited higher carotenoids content, while decreased in chlorophyll content because carotenoids are closely associated to chlorophyll due its supportive role in protection of chlorophyll. Stress circumstances could induce damage of photosynthetic compounds, thereby signaling for protection necessity. Consequently, it required a higher number of carotenoids to action via xanthophyll cycle to extenuating those kind of abnormality [33]. While Se was used as antioxidative molecules in plants exposed to other stressors including drought, UV-B, salinity, and even heavy metals, results revealed an increase in carotenoids content due to the Se oxidative stress protectant. Outcomes from a study on Se fortified to wheat exposed to drought stress confirmed this claim [34]. However, a reduction in $\beta$-carotene contents affected by Se was also observed in tomato fortified with Se (1 mg Se/L) [17]. It had been reported earlier that Se may down-regulate 
the expression of phytoene synthase which was a key step of carotenoid biosynthesis in rockcress (Arabidopsis), plant in Brassicaceae family [35]. Therefore, Se may down-regulate some enzymes or genes on carotenoids synthesis in tomato. Moreover, the lower amount of $\beta$-carotene in tomato may also be related to the ripening adjournment process induced by Se, which is influenced by the reduction of ethylene. Ethylene is the crucial hormone controlling ripening period of fruits. Since Se addition is formed a complex to methionine and converted to Se-Met which accumulate as organic Se in plant tissues. Therefore, Se bio-fortification reduces the level of free methionine, an important substrate of the ethylene biosynthesis and eventually lowering the production of ethylene [36].

\section{Phenolic compounds}

Phenolic compounds are the most plentiful secondary metabolites of plants that are essentially formed by the shikimate and phenylpropanoid pathways (Figure 2), with more than 8,000 known compounds, ranging from simple phenolic acids molecules to highly polymerize structures including flavonoids and tannins [37]. In plants, phenolic compounds typically work as protective elements against UV radiation, pathogens, and predators, while also contributing to some organoleptic properties of plant foods including color, taste, and flavor. Anthocyanins provided red and purple color to plants, with some phenolics giving a bitter and astringent taste. Moreover, they are required for the synthesis of lignin to support plants' cell wall structure. Therefore, these valuable compounds correspondingly deliver multiple biological effects including antioxidant activity, anti-inflammatory properties, and anti-carcinogenic properties [38]. Possible routes explaining how Se influence on plant phenolics include the following. First, it was previously reported that lightly exposed plants to stress conditions may stimulate the production of phenolic compounds because of their role as protectants in plants [39]. Goji leaves were demonstrated to have an improvement in chlorogenic acid content as affected of $5 \mathrm{mg} \mathrm{Se} / \mathrm{kg}$ of sodium selenite bio-fortification [21]. Furthermore, Se bio-fortified purple potatoes at low levels of Se (20 mg $\mathrm{Se} / \mathrm{L}$ ) were positively affected to raise phenolic acids content including chlorogenic acid, caffeic acid, and its derivatives, in addition to its impact on anthocyanin contents while higher levels revealed negative effects [40]. Additionally, a similar reason was explained for the effect of Se on flavonoid content that higher accumulation may result in plant's enhanced capacity to overcome stress conditions. For example, Se could enhance the level of naringin chalcone and kaemperol in tomato fruits [41], promote accumulation of catechin in Assam tea [42], and enhance anthocyanin content in wheat [27]. Responses of plants to stress can furthermore be observed from the level of proline in plant tissues which is regulated by shikimate pathway. Se stated to enhance the proline level in many plants including basil [30] and displays higher level of phenolic compounds, thereby confirming the induction of phenolic accumulation as response from stressors. Second, Sulfur (S) and Se are antagonists at similar routes of absorption by plant roots. The accumulation of one mineral, either $\mathrm{S}$ or nitrogen $(\mathrm{N})$, leads to the limitation of the other one as $\mathrm{N}$ and $\mathrm{S}$ is the major substrate for protein production. [43]. The addition of Se into plants is therefore highly interrelated to $\mathrm{N}$ metabolism by exert the accumulation of $\mathrm{N}$ compounds in plants comprise of amino acids, protein, and $\mathrm{N}$-secondary metabolites compounds. Amino acids including methionine, phenylalanine, tryptophan, and tyrosine are precursors of many intermediates compounds. In the case of phenolic compounds, phenylalanine is the major substrate for their biosynthesis. Third, Se 
may have additional effects on some enzymes responsible for phenolic compounds production in phenylpropanoids pathway (Figure 2). It has been reported that in order to stimulate the production of phenylalanine ammonia lyase (PAL), the enzyme for converting phenyl alanine to cinnamic acid is the first step in the biosynthesis of phenolic compounds through the activation of its expression. The hypothesis can be supported by studies in Se bio-fortified basil [30], in which total phenolic contents were raised in accordance to PAL activities.

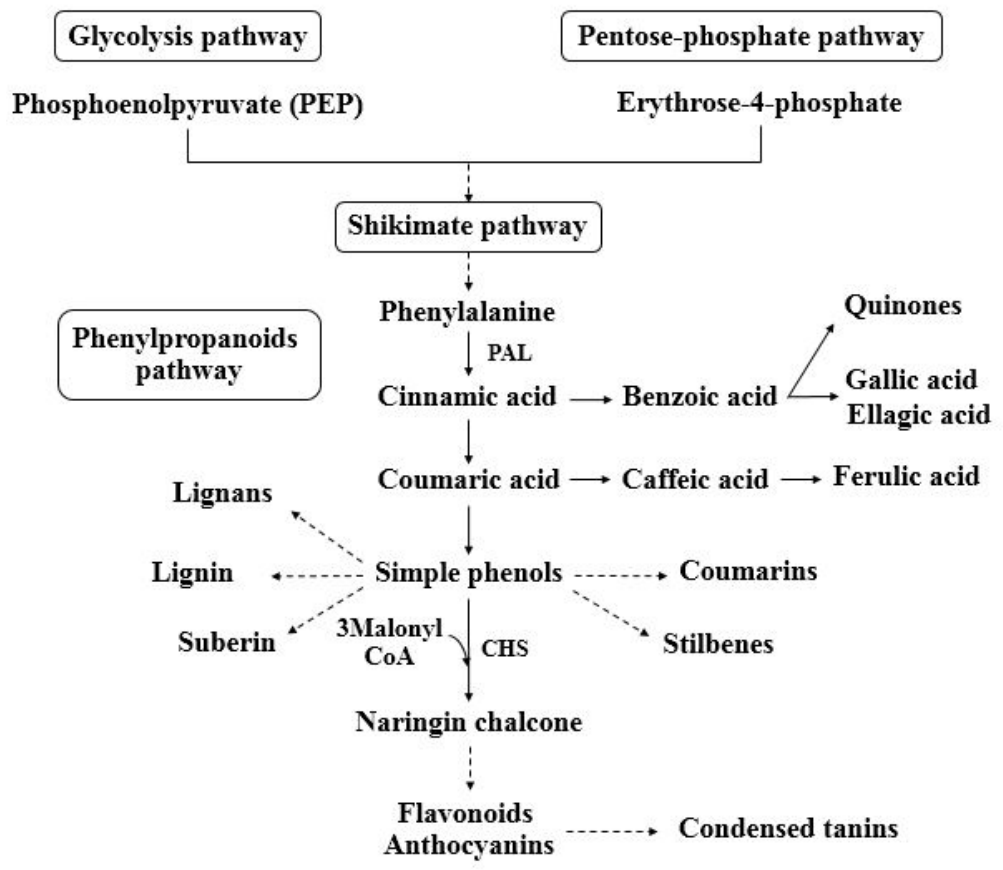

Figure 2. Biosynthesis of phenolic compounds in plants. Abbreviations: PAL, phenylalanine ammonia lyase; CHS, chalcone synthase.

\section{Glucosinolates (GLS)}

Glucosinolates are $\mathrm{S}$ and $\mathrm{N}$ containing secondary metabolites which exhibit anti-carcinogenic properties. They can be found in cruciferous plants (Brasisscaceae), including cauliflower, garden cress, cabbage, broccoli, or brussel sprouts. As Se and S are competitive at the same channels of the root membrane, Se bio-fortification may thereby effect on the level of GLS in plants. Exposing plants to low levels of Se may promote S uptake, which therefore may enhance the level of Smetabolites like GLS. As can be seen in a study conducted by Thiruvengadam and Chung (2015), selenium dioxide fortified to turnip could induce the production of some GLS derivatives including gloconasturtin, glucobrassicanapin, and 4-methylglucobrassicin [44]. On the other hand, high Se concentration can lead to reduction in GLS content. In broccoli shoots fed with sodium selenite there was a reduction in the level of GLS in addition to 4-methylsulphinylbutyl GLS [45]. GLS reduction mainly in the form of glucotropaeolin in garden cress sprouts were also observed after treatment with sodium selenate and sodium selenite 4 and $8 \mathrm{mg} \mathrm{Se} / \mathrm{L}$ [46]. A similar study on Se fortified broccoli also supported the fact that there was reduction of GLS in florets but not sprouts [47]. Reduction in total GLS in cruciferous plants may be explained by the influence of Se on the $\mathrm{S}$ assimilation pathway. The synthesis of seleno-amino acid instead of S-amino acid and further 
complexion to protein may interrupt GLS compounds production, thus reducing their level. Moreover, Se treatment has also been reported to up regulate genes responsible for GLS breakdown [48].

\section{Ascorbic acid and tocopherols}

Ascorbic acid, also known as vitamin $\mathrm{C}$, is the biomolecule that occurs in all the cell compartments of plants. Vitamin $\mathrm{C}$ acts in plants as an enzyme cofactor involved in photosynthesis and the control of regular growth. Moreover, vitamin $\mathrm{C}$ has been demonstrated to be the most potent antioxidant against radicals in water soluble phase prior to other defensive compounds like phenolics. Furthermore, vitamin $\mathrm{C}$ is able to reduce the oxidized tocopherol, the fat-soluble vitamin $\mathrm{E}$ which can exhibit oxidation process of lipid cellular membranes [49]. In response to stress induction by high levels of Se, ascorbic acid and tocopherol may possibly increase. A study on lettuce by Xue et al. (2001) suggested that Se promotes the production of tocopherol of senescent seedlings [24]. Experiment conducted on Se treated wheat also demonstrated a positive relationship between Se addition and glutathione content. As Se is a cofactor of GPx, it has been suggested that Se may increase ascorbic acid content through the stimulating effect on GSH, which may chemically reduce DHA to ascorbic acid via the ascorbate-glutathione cycle [50]. Ascorbic acid has a role in photosynthesis. Therefore, therefore ascorbic acid is greatly related to the growth and chlorophyll content of plants. Studies in sodium selenite bio-fortified to tomato seedling exposed parallel results with an increase of the amount of net photosynthetic rate and photosynthetic pigments which thereby results in increased amounts of ascorbate in plant tissues. However, at higher Se-treatment levels, there was evidence that in cactus pear treated with selenoferous soil the ascorbic acid content was reduced while the level of phenolic compounds tended to be higher [51]. This could be attributed to the primary defensive roles of ascorbic acid in plants. Ascorbic acid is utilized as electron donor to stabilize radicals in the plant system, but when its pool becomes low, plants might shift to other compounds including phenolics. Nevertheless, the trend in other plants may be different due to their different routes of defense systems.

\section{INFLUENCE OF SELENIUM ON PLANT BIOACTIVITIES} Antioxidant activities

In all type of cells, reactive oxygen species (ROS) are generated as a natural by-product of oxygen metabolism in the normal stage of life [52]. A balance between ROS and concomitant substances to scavenge them is necessary to maintain the proper physiological function in biological systems. However, when an excess amount of free radicals is produced, it may cause the injury to cells. In human nutrition, antioxidants are important to counteract the oxidisable biomolecules including lipids, proteins, and DNA and the promotion of prominence in health. Plants are like the other organisms when exposed to strain conditions, they have evolved mechanisms to manage the counterbalance. The endogenous antioxidant system of plants is compromised of enzymatic and non-enzymatic antioxidants. The enzymatic antioxidants included superoxide dismutase (SOD), glutathione peroxidase (GPx), catalase (CAT), peroxidase (POD), in addition to the enzymes of the ascorbate-glutathione cycle including ascorbate peroxidase (APx), monodehydroascrbate reductase (MDHAR), dehydroascorbate reductase (DHAR), and glutathione reductase (GR) while 
non-enzymatic antioxidants compounds included glutathione (GSH), ascorbic acid, carotenoids, tocopherols, and various phytochemicals, especially phenolic compounds. Se bio-fortification has the foremost effect on the antioxidant activities of plants, as Se is well-known as a core part of the GPx enzyme. Therefore, a large number of studies were addressed on the elimination of various kinds of stress owing to its efficiency in trigger enzymatic antioxidants system in plants. Lipid peroxidation has been recognized as a major cause of cellular injury which can be estimated from the concentration of the by-product in tissue extract called malondialdehyde (MDA) measuring from TBARS (Thiobarbutyric acid reactive species) assay [53]. As plants always initially manipulate these effects using enzymes, MDA and antioxidant enzymes content are the main biomarkers to observe plants' changes. Many researchers reported the helpfulness of Se in handling stress injury by enhancing the level of GPx, CAT, POD, and APx while plants are visible to UV-B stress [34], cold stress [16], drought stress [26], and salt stress [54, 55], even when exposed to heavy metals like lead $(\mathrm{Pb})$ and arsenic $(\mathrm{As})[56,57]$. Furthermore, Se addition was also proved to mitigate the lipid peroxidation by lowering MDA content in plant tissues [46, 55]. Possible explanation on this behavior can be suggested that the supplementation of Se supports GPx activity by being an enzyme complex. When the level of GPx was high in the system, the other enzymes which work on similar substrate $\left(\mathrm{H}_{2} \mathrm{O}_{2}\right)$ like CAT and APx were kept. Additionally, while the rate of $\mathrm{H}_{2} \mathrm{O}_{2}$ decomposition is high, SOD was highly prompted which resulted in its reduction. The relationship of antioxidant enzymes in plants is shown in Figure 3.

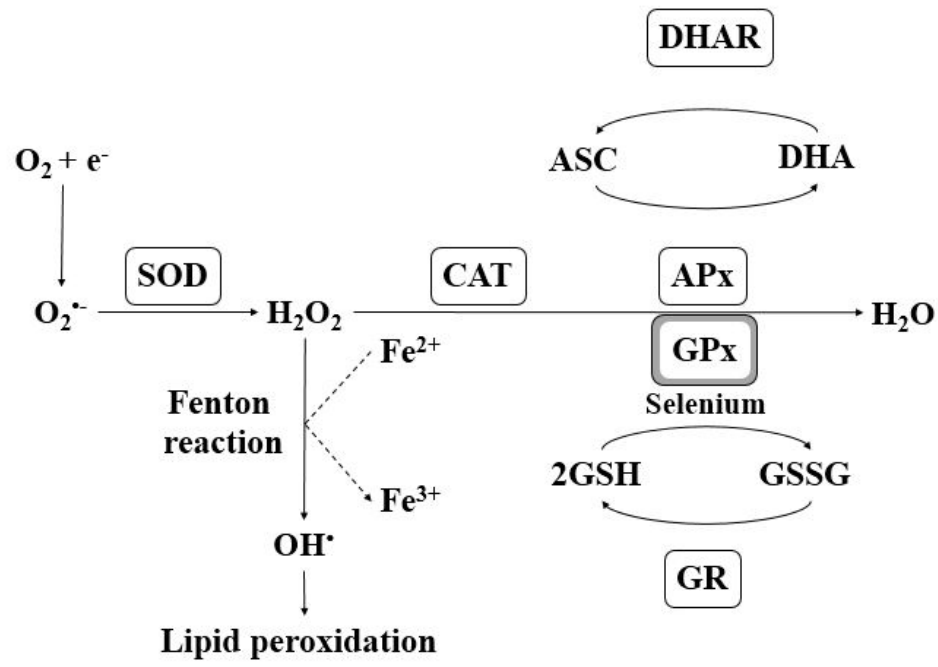

Figure 3. Antioxidant enzymes relationship in plants. Abbreviations: SOD, superoxide dismutase; CAT, catalase; APx, ascorbate peroxidase; ASC, ascorbic acid; DHA, dehydroascorbate; DHAR, dehydroascorbate reductase; GPx, glutathione peroxidase; GR, glutathione reductase; GSH, glutathione; GSSG, glutathionedisulfide.

Antioxidant activities in plants may also improve from the consequence of $\mathrm{Se}$ on phytochemicals components which have its function in electron or hydrogen atom donors. The well-known substance which potently works on this purpose were phenolic compounds. Many experiments have been done in vitro claiming the correlation between increased total phenolic content affected by Se to the progression of antioxidant activities in plants as measured by ABTS 
(2,2'-azino-bis-3-ethylbenzothiazoline-6-sulphonic acid) radical scavenging activities in cactus pear [51], DPPH (2,2-diphenyl-1-picrylhydrazyl) radical scavenging activities in pepper [58], FRAP (Ferric reducing antioxidant power) and FCA (Ferrous ion chelating activities) in green tea [59]. A similar result was also shown on an enhancement of DPPH, ABTS, and AAPH (2,2'-Azobis (2-amidinopropane) dihydrochloride) radical scavenging activities in Se bio-fortified rice shoots. Likewise, an improvement of carotenoids, tocopherol, and ascorbic acid affected by Se may also play some parts. However, only an appropriate amount of Se fortified may provide advantages. At high doses, it may exert toxic effects by acting as pro-oxidant, reducing yields and inducing metabolic disturbances. Additionally, Se itself may provide extra nutritional values to plants, especially while plants store Se in organic form. When consumed, Se supplement can promote the activity of GPx level in blood and liver which strengthens the protection ability of the body. Trials of rats fed with Se-broccoli floret extract which has been done by Charron et al. (2001) revealed an increase in red blood cell GPx activity significantly compared to rats fed with regular broccoli [45]. Another study in vivo on effect of Se-green tea fed to rats also demonstrated an increase in GPx activity together with the reduction blood MDA content better than sodium selenite [60]. This may additionally support the claim that the consumption of organic Se provides higher bioavailability in in vivo system.

\section{Anti-inflammatory properties}

Se disclosed anti-inflammatory properties in many studies in vitro and in vivo via various transduction pathways. It indicated the influence of cell signaling and inhibition of the activation of $\mathrm{NF \kappa B}$, which is the transcription factor that plays a role in response to inflammatory signaling [61]. Se may also enhance the number of $\mathrm{T}$ lymphocytes (T-cells) which play a role in cell mediated immunity, thereby provoking an anti-inflammatory effect [62]. Se bio-fortification within plants can be expected to provide anti-inflammatory effects both from Se itself and with its role to trigger the bioactive compounds that have the potential role of suppressing inflammation such as flavonoids. However, only a few reports noticed this issue. The results obtained from Tyszka-Czochara et al. (2016) showed that the extract of amaranth sprouts grown in sodium selenite can significantly decrease in IL-6 concentration in the medium of lipopolysaccharides (LPS) activated RAW 264.7 cell without toxicity compared to the control [63]. However, the effect on the transcription factor NFאB in cells was not different. Research on plants rich in Se which possess anti-inflammatory properties still needs to be supported by additional evidence.

\section{Anti-tumor and anti-cancer properties}

Along with numerous studies related to the health benefits of Se, the powerful effect of Se as antitumor and anti-cancer substance has been widely spread. The possible mechanisms of Se to inhibit cancer included the reduction of DNA damage [64], reduction of oxidative stress, suppression of inflammation [65], and enhancement of response of the immune system by increasing the numbers of cytotoxic lymphocytes and natural-killer cells [9]. Moreover, nutrigenetic researchers discovered that seleno-methionine, organic Se can activate tumor suppressor protein p53 gene which can inhibit the proliferation and induce the early apoptosis of cancer cells [66,67]. Reciprocal to other bioactivities, Se bio-fortified plants were likely to employ benefits from both 
the ability of high Se to work as part of enzyme complex and the induction of Se on plant phytochemicals. In vitro study of ethanolic extract of green tea applied with foliar application of Se possessed the outcomes on inhibiting the growth of cancer cells included HeLa (human cervix adenocarcinoma), A549 (human lung carcinoma), and Hep G2 (human liver hepatocellular carcinoma) cell lines [68]. This study suggested responsibility of Se to trigger the production of tea polyphenols, especially epigallocatechin gallate (EGCG) which can promote higher antioxidant activities and anti-cancer properties.

Se-accumulating plants including Allium and Brassica plants that can accumulate high content of Se are thereby the most remarkable group of plants to be supplied by Se to yield natural products containing extraordinary anti-cancer ability. High Se garlic was reported to significantly suppress the development of lesions and adenocarcinomas formation in the mammary gland of carcinogen treated rats better than Se-yeast due to the high content of $\gamma$-glutamyl-Se-methylselenocysteine which is believed to be efficient against cancer cells [69]. The works of Se enrichment in broccoli has been tested on anti-cancer potential and showed a $50 \%$ reduction in aberrant crypts in colon of mice [70], and also reduced the incidence of mice mammary tumors and tumor number [71]. In the same way, Se fortified ramp supported ability of Se-plant to reduce the chemically induced mammary tumor in rat. Additionally, another study suggested significant reduction in $\beta$ glucuronidase bacterial enzyme activity which administrated rats with Se enrich green tea drink compared with the rats fed with water only [72]. Because the $\beta$-glucuronidase enzyme is considered to be one of the enzymes that increases the risk for colorectal cancer, it thereby reveals alternative roles of Se to reduce incidence of colon cancer.

\section{Prebiotic properties}

Prebiotics are non-digestible food ingredients that intend to stimulate the growth of probiotic bacteria, the viable beneficial microorganisms in human gut [73]. Se fortified green tea has been stated by Molan et al. (2009) for the first time to contain prebiotic effects by promoting the growth of Lactobacillus rhamnosus and Bifidobacterium breve when added in MRS broth [59]. Moreover, their animal model study investigated the population size of probiotic and pathogenic bacteria in the cecum of rat after oral administration with aqueous extracted of Se containing green tea compared to the regular Chinese green tea [72]. Results revealed an increase in Lactobacillus and Bifidobacteria species with a significant reduction of the number of bacteroides and clostridial bacteria. While the control showed a slight increase in the number of probiotics, the effect was not significant. Se enriched green tea is now claimed to be a good prebiotic food underlying the compromise of Se to promote phenolic compounds production in green tea. High levels of phenolic compounds thereby offers higher antioxidant effects and modulation of oxidative stress in the medium which provides a better environment for the multiplication of probiotics, in addition to somehow inhibiting pathogens. Nonetheless, only the studies on Se-green tea were reported. Further work need to be claimed on its noteworthy effects.

\section{CONCLUSION}

Se bio-fortification regards the influence that plants produce in many ways as this strategy originates from the deficiency problem. The increased content of Se after bio-fortification is not 
only an issue that matters to bioactivities of plants, but also stimulates the production of phytochemicals. To the best of our knowledge, this review concluded that Se bio-fortified into plant resulted in both negative and positive behaviors of plants. Principally, advantageous effects of Se occurs when plants are induced in the early stage of stress. At this stage, plants attempt to overwhelm the strain condition from the production of protective components. Se at low dosages tended to improve the overall nutritional quality of plants while higher levels were toxic. The suitable exogenous concentration of Se bio-fortified to individual plants is thereby the most critical factor to provide desired effects from an improvement of bioactive compounds including chlorophyll, carotenoids, phenolic compounds, glucosinolates, and ascorbic acids. Additionally, Se supplementation positively influences the biological properties of plants including antioxidant, anti-inflammation, anti-cancer, and prebiotic properties. Focus research on Se application in specific plant species is yet required in order to use this strategy in unrestricted approach. Se biofortification can now be unquestionably claimed to be a good strategy to improve the nutritional values of plants.

Abbreviations: TPC: Total extractable phenolic content; DPPH: 1,1-diphenyl-2-picryl-hydrazyl; ABTS: 2,2'-azino-bis(3-ethylbenzthiazoline-6-sulfonic acid); FRAP: Ferric reducing antioxidant power; FCA: Ferric ion chelating activity; AAPH: (2,2'-Azobis (2-amidinopropane) dihydrochloride) radical scavenging activities;SOD: superoxide dismutase; CAT: catalase; APx, ascorbate peroxidase; ASC: ascorbic acid; DHA: dehydroascorbate; DHAR: dehydroascorbatereductase; GPx: glutathione peroxidase; GR: glutathione reductase; GSH: glutathione; GSSG: glutathionedisulfide; Se-Proteins: seleno-proteins; Se-Met: selenomethionine; Se-Cys:seleno-cysteine; Se-Methyl-SeCys:seleno-methyl-selenocysteine; DMSe: dimethyl-selenide; DMDSe: dimethyl-diselenide,PAL: phenylalanine ammonia lyase; CHS: chalcone synthase.

Competing interests: The authors have declared that no competing interests exist.

Author's contributions: Rattanamanee Chomchan, $\mathrm{PhD}$ candidate, is the major investigator, collecting all the relevant studies, performed the analysis and writing the manuscript. Sunisa Siripongvutikorn, $\mathrm{PhD}$, is an Assistant Professor of food technology. She provided vision and conceptualization of this review. She was contributing to the verification of manuscript. Panupong Puttarak, PhD, is a doctor of pharmaceutical sciences. He is a research coordinator who provided remarks, suggestion as well as verified the correction of manuscript.

Acknowledgements: Authors would like to acknowledge scholarship from Thailand's Education Hub for Southern Region of ASEAN Countries (TEH-AC) and Prince of Songkla University research grant to pursue graduate studies. Authors ultimately assemble to previous published literatures related to the influence of Se bio-fortification on phytochemicals and bioactivities of countless plant. Though we finest tried to combine all the relevant studies, an apology is regarded for some neglect studies. 


\section{REFERENCES:}

1. Balandrin, M.F., Klocke, J.A., Wurtele,E.S. and Bollinger, W.H. Natural plant chemicals: sources of industrial and medicinal materials. Science 1985; 228:1154-1160.

2. Thomasset, S.C., Berry, D.P., Garcea, G., Marczylo, T., Steward, W.P. and Gescher, A.J. Dietary polyphenolic phytochemicals - promising cancer chemopreventive agents in humans? A review of their clinical properties. Int J Cancer 2007; 120:451-458.

3. Dembinska-Kiec, A., Mykkänen, O., Kiec-Wilk, B. and Mykkänen, H. Antioxidant phytochemicals against type 2 diabetes. Brit J Nutr 2008; 99:ES109-ES117.

4. González-Castejón, M. and Rodriguez-Casado, A. Dietary phytochemicals and their potential effects on obesity: a review. Pharmacol Res 2011; 64:438-455.

5. Randhir, R., Lin, Y.T. and Shetty, K. Phenolics, their antioxidant and antimicrobial activity in dark germinated fenugreek sprouts in response to peptide and phytochemical elicitors. Asia Pac J Clin Nutr 2004; 13:295-307.

6. Radman, R., Saez, T., Bucke, C. and Keshavarz, T. Elicitation of plants and microbial cell systems. Biotechnol Appl Biochem 2003; 37:91-102.

7. Dörnenburg, H. and Knorr, D. Strategies for the improvement of secondary metabolite production in plant cell cultures. Enzyme Microb Technol 1995; 17: 674-684.

8. White, P.J. and Broadley, M.R. Biofortifying crops with essential mineral elements. Trends Plant Sci 2005; 10: 586-593.

9. Kiremidjian-Schumacher, L., Roy, M.,Wishe, H.I., Cohen, M.W. and Stotzky, G. Supplementation with selenium augments the functions of natural killer and lymphokineactivated killer cells. Biol Trace Elem Res 1996; 52:227-239.

10. Rotruck, J., Pope, A., Ganther, H., Swanson, A., Hafeman, D.G. and Hoekstra, W. Selenium: biochemical role as a component of glutathione peroxidase. Science 1973; 179:588-590.

11. Dumont, E., Vanhaecke, F. and Cornelis, R. Selenium speciation from food source to metabolites: a critical review. Anal Bioanal Chem 2006; 385:1304-1323.

12. Rayman, M.P. Selenium and human health. Lancet 2012; 379:1256-1268.

13. Ellis, D.R. and Salt, D.E. Plants, selenium and human health. Curr Opin Plant Biol 2003; 6:273-279.

14. Zayed, A., Lytle, C.M. and Terry, N. Accumulation and volatilization of different chemical species of selenium by plants. Planta 1998; 206:284-292.

15. Ganther, H., Levander, O. and Baumann, C. Dietary control of selenium volatilization in the rat. J Nutr 1966; 88:55-60.

16. Chen, C.C. and Sung, J.M. Priming bitter gourd seeds with selenium solution enhances germinability and antioxidative responses under sub-optimal temperature. Physiol Plant 2001; 111:9-16.

17. Pezzarossa, B., Rosellini, I., Borghesi, E., Tonutti, P. and Malorgio, F. Effects of Seenrichment on yield, fruit composition and ripening of tomato (Solanum lycopersicum) plants grown in hydroponics. Sci Hort 2014; 165:106-110. 
18. Eurola, M., Ekholm, P., Ylinen, M., Koivistoinen, P. and Varo, P. Effects of selenium fertilization on the selenium content of selected Finnish fruits and vegetables. Acta Agr Scand 1989; 39:345-350.

19. Li, F., Wang, F., Yu, F., Fang, Y., Xin, Z., Yang, F., Xu, J., Zhao, L. and Hu, Q. In vitro antioxidant and anticancer activities of ethanolic extract of selenium-enriched green tea. Food Chem 2008; 111:165-170.

20. Lichtenthaler, H.K. Chlorophyll and carotenoids: pigments of photosynthetic biomembranes. Method Enzymol 1987; 148:350-382.

21. Dong, J.Z., Wang, Y., Wang, S.H., Yin, L.P., Xu, G.J., Zheng, C., Lei, C. and Zhang, M.Z. Selenium increases chlorogenic acid, chlorophyll and carotenoids of Lycium chinense leaves. J Sci Food and Agr 2013; 93:310-315.

22. Lavu, R.V.S., De Schepper, v., Steppe, K., Majeti, P.N., Tack, F. and Du Laing, G. Use of selenium fertilizers for production of Se-enriched Kenaf (Hibiscus cannabinus): Effect on Se concentration and plant productivity. J Plant Nutr Soil Sci 2013; 176:634-639.

23. Hawrylak-Nowak, B. Comparative effects of selenite and selenate on growth and selenium accumulation in lettuce plants under hydroponic conditions. Plant Growth Reg 2013; 70:149-157.

24. Xue, T., Hartikainen, H. and Piironen, V. Antioxidative and growth-promoting effect of selenium on senescing lettuce. Plant Soil 2001; 237:55-61.

25. Padmaja, K., Prasad, D. and Prasad, A. Selenium as a novel regulator of porphyrin biosynthesis in germinating seedlings of mung bean (Phaseolus vulgaris). Biochem Int 1990; 22:441-446.

26. Yao, X., Chu, J. and Wang, G. Effects of selenium on wheat seedlings under drought stress. Biol Trace Elem Res 2009; 130:283-290.

27. Chu, J., Yao, X. and Zhang, Z. Responses of wheat seedlings to exogenous selenium supply under cold stress. Biol Trace Elem Res 2010; 136:355-363.

28. Seppänen, M., Turakainen, M. and Hartikainen, H. Selenium effects on oxidative stress in potato. Plant Sci 2003; 165:311-319.

29. Padmaja, K., Prasad, D. and Prasad, A. Effect of selenium on chlorophyll biosynthesis in mung bean seedlings. Phytochem 1989; 28:3321-3324.

30. Ardebili, Z.O., Ardebili, N.O., Jalili, S. and Safiallah, S. The modified qualities of basil plants by selenium and/or ascorbic acid. Turkish J Bot 2015; 39:401-407.

31. Meyer, S., Cerovic, Z., Goulas, Y., Montpied, P., Demotes-mainard, S., Bidel, L., Moya, I. and Dreyer, E. Relationships between optically assessed polyphenols and chlorophyll contents, and leaf mass per area ratio in woody plants: a signature of the carbon-nitrogen balance within leaves? Plant Cell Environ 2006; 29:1338-1348.

32. Armstrong, G.A. and Hearst, J.A. Carotenoids 2: Genetics and molecular biology of carotenoid pigment biosynthesis. FASEB J 1996; 10:228-237.

33. Li, G., Wan, S., Zhou, J., Yang, Z. and Qin, P. Leaf chlorophyll fluorescence, hyperspectral reflectance, pigments content, malondialdehyde and proline accumulation responses of castor bean (Ricinus communis L.) seedlings to salt stress levels. Ind Crop Prod 2010; 31:13-19. 
34. Yao, X., Chu, J. and Ba, C. Antioxidant responses of wheat seedlings to exogenous selenium supply under enhanced Ultraviolet-B. Biol Trace Elem Res 2010; 136:96-105.

35. Sams, C.E., Panthee, D.R., Charron, C.S., Kopsell, D.A. and Yuan, J.S. Selenium regulates gene expression for glucosinolate and carotenoid biosynthesis in Arabidopsis. J Am Soc Hort Sci 2010; 136:23-34.

36. Tapiero, H., Townsend, D. and Tew. K. The antioxidant role of selenium and selenocompounds. Biomed Pharmcother 2003; 57:134-144.

37. Ramawat, K.G. and Dass, S. Herbal drugs: ethnomedicine to modern medicine. Berlin, Springer, 2009.

38. Dai, J. and Mumper, R.J. Plant phenolics: extraction, analysis and their antioxidant and anticancer properties. Molecules 2010; 15:7313-7352.

39. Winkel-Shirley, B. Biosynthesis of flavonoids and effects of stress. Curr Opin Plant Biol 2002; 5:218-223.

40. Lei, C., Ma, Q., Tang, QY., Ai, X.R., Zhou, Z., Yao, L., Wang, Y., Wang, Q. and Dong, J.Z. Sodium selenite regulates phenolics accumulation and tuber development of purple potatoes. Sci Hort 2014; 165:142-147.

41. Schiavon, M., dall'Acqua, S., Mietto, A.,Pilon-Smits, E.A., Sambo, P., Masi, A. andMalagoli. M. Selenium fertilization alters the chemical composition and antioxidant constituents of tomato (Solanum lycopersicon L.). J Agr Food Chem 2013; 61:1054210554.

42. Sae-Lee, N., Kerdchoechuen, O. and Laohakunjit, N. Chemical qualities and phenolic compounds of Assam tea after soil drench application of selenium and aluminium. Plant Soil 2012; 356:381-393.

43. Barney, Jr.P. and Bush, L. Interaction of nitrate and sulfate reduction in tobacco: Influence of availability of nitrate and sulfate. J Plant Nutr 1985; 8:505-515.

44. Thiruvengadam, M. and Chung, I.M. Selenium, putrescine, and cadmium influence healthpromoting phytochemicals and molecular-level effects on turnip (Brassica rapa ssp. rapa). Food Chem 2015; 173:185-193.

45. Charron, C.S., Kopsell, D.A., Randle, W.M. and Sams, C.E. Sodium selenate fertilisation increases selenium accumulation and decreases glucosinolate concentration in rapidcycling Brassica oleracea. J Sci Food Agr 2001; 81:962-966.

46. Frias, J., Gulewicz, P., Martinez-Villaluenga, C., Peñas, E., Piskula, M.K., Kozlowska, H., Ciska, E., Gulewicz, K., andVidal-Valverde, C. Changes in nutritional value and cytotoxicity of garden cress germinated with different selenium solutions. J Agr Food Chem 2010; 58:2331-2336.

47. Ávila, F.W., Faquin, V., Yang, Y., Ramos, SJ., Guilherme, L.R.G., Thannhauser, T.W. and Li, L. Assessment of the anticancer compounds Se-methylselenocysteine and glucosinolates in Se-biofortified broccoli (Brassica oleracea L. var. italica) sprouts and florets. J Agr Food Chem 2013; 61:6216-6223.

48. Van Hoewyk, D., Takahashi, H., Inoue, E., Hess, A., Tamaoki, M. and Pilon-Smits, E.A. Transcriptome analyses give insights into selenium-stress responses and selenium tolerance mechanisms in Arabidopsis. Physiol Plant 2008; 132:236-253. 
49. Bendich, A., Machlin, L., Scandurra, O., Burton, G. and Wayner, D. The antioxidant role of vitamin C. Adv Free Rad Biol Med 1986; 2:419-444.

50. Ibrahim, H.M. Selenium pretreatment regulates the antioxidant defense system and reduces oxidative stress on drought-stressed wheat (Triticum aestivum L.) plants. Asian J Plant Sci 2014; 13:120-128.

51. Bañuelos, G.S., Stushnoff, C., Walse, S.S., Zuber, T., Yang, S.I., Pickering, I.J. and Freeman, J.L. Biofortified, selenium enriched, fruit and cladode from three Opuntia Cactus pear cultivars grown on agricultural drainage sediment for use in nutraceutical foods. Food Chem 2012; 135:9-16.

52. Devasagayam, T., Tilak, J., Boloor, K., Sane, K.S., Ghaskadbi, S.S. and Lele, R. Free radicals and antioxidants in human health: current status and future prospects. J Assoc Phy India 2004; 52:794-804.

53. Largillière, C. and Mélancon, S.B. Free malondialdehyde determination in human plasma by high-performance liquid chromatography. Anal Biochem 1988; 170:123-126.

54. Hasanuzzaman, M., Hossain, M.A. and Fujita, M. Selenium-induced up-regulation of the antioxidant defense and methylglyoxal detoxification system reduces salinity-induced damage in rapeseed seedlings. Biol Trace Elem Res 2011; 143:1704-1721.

55. KeLing, H., Ling, Z., JiTao, W. and Yang, Y. Influence of selenium on growth, lipid peroxidation and antioxidative enzyme activity in melon (Cucumis melo L.) seedlings under salt stress. Acta Soc Bot Pol 2013; 82:193-197.

56. Kumar, A., Singh, R.P., Singh, P.K., Awasthi, S., Chakrabarty, D., Trivedi P.K. and Tripathi, R.D. Selenium ameliorates arsenic induced oxidative stress through modulation of antioxidant enzymes and thiols in rice (Oryza sativa L.). Ecotoxicol 2014; 23:11531163.

57. Mroczek-Zdyrska, M. and Wójcik, M. The influence of selenium on root growth and oxidative stress induced by lead in Vicia faba L. minor plants. Biol Trace Elem Res 2012; 147:320-328.

58. Mozafariyan, M., Shekari, L., Hawrylak-Nowak, B. and Kamelmanesh, M.M. Protective role of selenium on pepper exposed to cadmium stress during reproductive stage. Biol Trace Elem Res 2014; 160:97-107.

59. Molan, A., Flanagan, J., Wei, W. and Moughan, P. Selenium-containing green tea has higher antioxidant and prebiotic activities than regular green tea. Food Chem 2009; 114 : 829-835.

60. Hu, Q., Pan, G. and Zhu, J. Effect of fertilization on selenium content of tea and the nutritional function of Se-enriched tea in rats. Plant Soil 2002; 238:91-95.

61. Duntas, L. Selenium and inflammation: underlying anti-inflammatory mechanisms. Horm Metab Res 2009; 41:443-447.

62. Wood, S.M., Beckham, C., Yosioka, A., Darban, H. and Watson, R.R. $\beta$-Carotene and selenium supplementation enhances immune response in aged humans. Int Med 2000; 2: 85-92.

63. Tyszka-Czochara, M., Pasko, P., Zagrodzki, P., Gajdzik, E., Wietecha-Posluszny, R. and Gorinstein, S. Selenium supplementation of amaranth sprouts influences betacyanin 
content and improves anti-Inflammatory properties via NFKB in murine RAW 264.7 macrophages. Biol Trace Elem Res 2016; 169:320-330.

64. Waters, D.J., Shen, S., Glickman, L.T., Cooley, D.M., Bostwick, D.G., Qian, J., Combs, G.F. and Morris, J.S. Prostate cancer risk and DNA damage: translational significance of selenium supplementation in a canine model. Carcinogenesis 2005; 26:1256-1262.

65. Rayman, M.P. The importance of selenium to human health. Lancet 2000; 356:233-241.

66. Chen, T. and Wong, Y.S. Selenocystine induces caspase-independent apoptosis in MCF-7 human breast carcinoma cells with involvement of p53 phosphorylation and reactive oxygen species generation. Int J Biochem Cell Biol 2009; 41:666-676.

67. Smith, M., Lancia, J., Mercer, T. and Ip, C. Selenium compounds regulate p53 by common and distinctive mechanisms. Anticancer Res 2003; 24:1401-1408.

68. Li, H.F., McGrath, S.P. and Zhao, F.J. Selenium uptake, translocation and speciation in wheat supplied with selenate or selenite. New Phytol 2008; 178:92-102.

69. Ip, C., Birringer, M., Block, E., Kotrebai, M., Tyson, J.F., Uden, P.C. and Lisk, D.J. Chemical speciation influences comparative activity of selenium-enriched garlic and yeast in mammary cancer prevention. J Agr Food Chem 2000; 48:2062-2070.

70. Finley, J.W., Davis, C.D. and Feng, Y. Selenium from high selenium broccoli protects rats from colon cancer. J Nutr 2000; 130:2384-2389.

71. Finley, J.W. Reduction of cancer risk by consumption of selenium-enriched plants: enrichment of broccoli with selenium increases the anticarcinogenic properties of broccoli. J Med Food 2003; 6:19-26.

72. Molan, A.L., Liu, Z. and Tiwari, R. The ability of green tea to positively modulate key markers of gastrointestinal function in rats. Phytother Res 2010; 24:1614-1619.

73. Schrezenmeir, J. and de Vrese, M. Probiotics, prebiotics, and synbiotics-approaching a definition. Am J Clin Nutr 2001; 73:361s-364s. 